\title{
Cognitive and Motor Aspects of Parkinson's Disease Associated with Dysphagia
}

\author{
Ji Sun Kim, Jinyoung Youn, Mee Kyung Suh, Tae-Eun Kim, Juhee Chin, \\ Suyeon Park, Jin Whan Cho
}

\begin{abstract}
Background: Dysphagia is a common symptom and an important prognostic factor in Parkinson's disease (PD). Although cognitive and motor dysfunctions may contribute to dysphagia in patients with PD, any specific association between such problems and swallowing functions is unclear. Here, we examined the potential relationship between cognitive/motor components and swallowing functions in PD. We evaluated the contributions of cognition and motor function to the components of swallowing via video fluoroscopic swallowing (VFS) experiments. Methods: We prospectively enrolled 56 patients without dementia having PD. Parkinson's disease severity was assessed by the Unified Parkinson's Disease Rating Scale (UPDRS). All participants received neuropsychological tests covering general mental status, visuospatial function, attention, language, learning and memory, and frontal executive function. The well-validated "modified barium swallow impairment profile" scoring system was applied during VFS studies to quantify swallowing impairments. Finally, correlations between neuropsychological or motor functions and impairment in swallowing components were calculated. Results: The most significant correlations were found between the frontal/executive or learning/memory domains and the oral phase of swallowing, though a minor component of the pharyngeal phase correlated with frontal function as well. Bradykinesia and the UPDRS total score were associated with both the pharyngeal and oral phases. Conclusions: Our findings suggest that cognitive dysfunctions are associated with the oral phase of swallowing in patients with early stage PD while the severity of motor symptoms may be associated with overall swallowing function.
\end{abstract}

RÉSUMÉ: Aspects cognitifs et moteurs de la maladie de Parkinson qui sont associés à la dysphagie. Contexte $:$ La dysphagie est un symptôme fréquent de la maladie de Parkinson (MP) et constitue un facteur de prédiction important dans cette maladie. Bien que les dysfonctions cognitives et motrices puissent contribuer à la dysphagie chez les patients atteints de la MP, une association spécifique entre ces problèmes et la déglutition demeure incertaine. Nous avons examiné une relation potentielle entre les composantes cognitives/motrices et la déglutition dans la MP. Nous avons évalué la contribution de la fonction cognitive et de la fonction motrice aux différentes composantes de la déglutition au moyen de la vidéofluoroscopie (DVF). Méthode : Nous avons recruté prospectivement 56 patients atteints de la MP, sans démence. La sévérité de la MP a été évaluée au moyen de la Unified Parkinson's Disease Rating Scale (UPDRS). Tous les participants ont subi des tests neuropsychologiques évaluant l'état mental général, la fonction visuospatiale, l'attention, le langage, l'apprentissage, la mémoire ainsi que les fonctions exécutives frontales. Le système « modified barium swallow impairment profile », un système d'évaluation validé, a été utilisé au cours des études de DVF pour quantifier l'atteinte de la fonction de déglutition. De plus, nous avons calculé les corrélations entre les fonctions neuropsychologiques ou motrices et l'atteinte des composantes de la déglutition. Résultats : Les corrélations les plus significatives observées étaient celles entre les domaines frontaux/exécutifs ou apprentissage/mémoire et la phase orale de la déglutition, bien qu'une composante mineure de la phase pharyngée ait été également corrélée à la fonction frontale. La bradykinésie et le score total au UPDRS étaient associés avec les phases pharyngée et orale. Conclusions : Selon nos observations, les dysfonctions cognitives sont associées à la phase orale de la déglutition chez les patients en phase précoce de la MP et la sévérité des symptômes moteurs pourrait être associée à la globalité de la fonction de déglutition.

Keywords: Dysphagia, neuropsychology, oral phase, Parkinson's disease

doi: $10.1017 / \operatorname{cjn} .2015 .304$

Can J Neurol Sci. 2015; 42: 395-400

Dysphagia affects the quality of life and increases the risk of aspiration pneumonia, a leading cause of death in Parkinson's disease (PD). ${ }^{1,2}$ Prevalence rates for swallowing dysfunction in
PD vary widely, ${ }^{3}$ though a recent meta-analysis noted that such patients are three times more likely to have swallowing problems than healthy controls. ${ }^{4}$ Furthermore, studies using objective tests,

From the Department of Neurology (JSK), Department of Biostatistics (SP), Soonchunhyang University Hospital, Soonchunhyang University School of Medicine, Seoul, Korea; Department of Neurology (JY, MKS, JC, JWC), Sungkyunkwan University School of Medicine; Neuroscience Center (JY, JWC), Samsung Medical Center, Seoul; Department of

Neurology (TK), Gwangmyeong Seongae Hospital, Gwangmyeong, Korea.

ReCeived May 21, 2015. Final Revisions Submitted July 15, 2015.

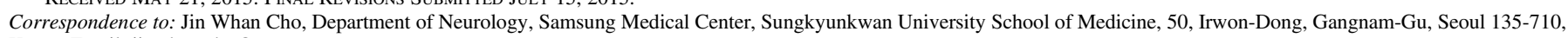
Korea. Email: jinwhan.cho@samsung.com 
including the modified barium swallow (MBS) test, report even higher percentages of swallowing dysfunction than those reported through subjective self-observation in patients with PD. ${ }^{4-6}$ Few studies have explored the contributing factors for PD-related dysphagia, but one did suggest that gender, age, disease duration, and dementia might all contribute. ${ }^{7}$ However, several reports indicate that dysphagia does not show a clear correlation with either disease duration or motor symptom severity. ${ }^{5,8,9}$ Despite its importance, the exact nature and pathophysiology underlying PD-related dysphagia remains unclear.

Swallowing behavior involves a set of complex neuromuscular activities comprising oral, pharyngeal, and esophageal phases, each of which is controlled by a different neurological mechanism. ${ }^{10}$ The pharyngo-esophageal swallowing sequence occurs via a brainstemdriven basic reflex, which utilizes sensory input from the oropharynx and esophagus. Because PD-related dysphagia is attributed to brainstem pathology, ${ }^{11}$ research about swallowing dysfunction has focused mainly on the functions of brainstem swallowing centers, including the dorsal motor nucleus of the glossopharyngeus, the vagus nerves, and the nucleus of the solitary tract. ${ }^{12,13}$

However, swallowing behavior is not under exclusive control of the brainstem. Rather, the initiation of swallowing has voluntary components that require integration of multiple cerebral cognitive functions. Recent clinical, electrophysiological, and neuroimaging studies have found that not only the brainstem, but also the basal ganglia and cerebral cortical circuits, affect the modulation of swallowing functions. ${ }^{14,15}$ Although all stages of swallowing may be affected by cortical functions, the oral phase of swallowing in patients with PD may be particularly affected. $5,16,17$ The oral phase can be subdivided into the oral preparatory phase, in which the bolus is actively manipulated and masticated, and the oral transport phase, in which lingual propulsive forces transport the bolus efficiently into the pharynx. ${ }^{18,19}$ These phases increase cognitive demand through the need for attention and volitional effort for planning and organized movement. ${ }^{20}$ However, few studies have explored the relationship between dysphagia and cognitive function.

The primary goal of this study was to evaluate the undefined relationship between cognitive function and dysphagia in PD using videofluoroscopic swallowing (VFS) studies in tandem with detailed neuropsychological tests assessing cognition, and studies assessing motor symptom severity.

\section{METHODS}

\section{Patients}

Subjects were recruited from 148 consecutive patients with PD seen at the Samsung Medical Center. These patients were positively diagnosed as PD according to the criteria of the UK Parkinson's Disease Society Brain Bank in the period from January, 2010 to January, 2012. Sixty-seven patients refused to participate. Twentyfive subjects were excluded due to (1) cognitive decline, defined as a score of $<24$ on the Korean version of the Mini-Mental State Examination (K-MMSE) ${ }^{21}$; (2) their inability to complete the questionnaires due to severe motor deficits; (3) a history of dysphagia prior to PD symptom onset; and (4) comorbidity associated with swallowing changes. We thus enrolled 56 patients with PD who agreed to participate the study. Patients were treated with levodopa and their medication states were kept stable throughout the study period. All VFS studies were performed during patients' "on" state, within two hours following levodopa intake. This study was approved by the Samsung Medical Center Ethics Committee, and written informed consent was obtained from each participant.

\section{Clinical Assessment}

Demographic information from all patients was gathered, including age, gender, duration of disease, and education. All patients underwent clinical interviews and neurological examinations by an experienced neurologist (J.W.C.). All patients were scored on the motor portion of the Unified Parkinson's Disease Rating Scale (UPDRS Part III) and Hoehn and Yahr (H \&Y) stages. Unified Parkinson's Disease Rating Scale scores were divided into subscores for tremor, rigidity, bradykinesia, and axial impairment according to the DATATOP factorial division. ${ }^{22}$ The sum of UPDRS items 20 and 21 was used for the tremor score, item 22 for the rigidity score, the sum of items $24,25,26$, and 31 for the bradykinesia score, and that of items 27-30 for the axial impairment score (arising from a chair, posture, gait, and postural stability, respectively).

\section{Neuropsychological Assessment}

All patients and controls underwent the Seoul Neuropsychological Screening Battery (SNSB) that includes standardized and validated tests of a variety of cognitive functions. ${ }^{23}$ The SNSB is composed of subcategories for testing of general mental status, attention, language and related functions, visuospatial function, memory, and frontal lobe/executive functions. Detailed tests included the K-MMSE, Clinical Dementia Rating (CDR), Digit Span Test, Korean version of the Boston Naming Test $(\mathrm{K}-\mathrm{BNT}){ }^{24}$ Seoul Verbal Learning Test (SVLT), semantic and phonemic Controlled Oral Word Association Test (COWAT), and Stroop word and color reading test. The clock-drawing test was used to test visuospatial function instead of the Rey-Osterrieth Complex Figure in consideration of potential motor dysfunction.

\section{Measurement of Swallowing Impairment via the VFS Study}

To quantify the overall physiological swallowing impairment, the Modified Barium Swallow Impairment Profile (MBSImP) was used. $^{25}$ This validated and reliable tool utilizes an ordinal scoring scheme that permits quantification of impairment of the oral, pharyngeal, and esophageal components of swallowing. The oral phase is composed of lip closure, hold position/tongue control, bolus preparation/mastication, bolus transport/lingual motion, oral residue, and initiation of the pharyngeal swallow. The pharyngeal phase includes soft palate elevation, laryngeal elevation, anterior hyoid motion, epiglottic movement, laryngeal closure, the pharyngeal stripping wave, pharyngeal contraction, pharyngoesophageal segment opening, tongue base retraction, and pharyngeal residue. The esophageal phase includes esophageal clearance, as determined in the upright position. We also assessed the occurrence of penetration and aspiration. The overall oral and pharyngeal stage scores represent the sums of their respective swallowing components.

Implementation of the MBSImP requires a judgment or overall impression score for each component across all swallow attempts during the MBS test. Modified Barium Swallow Impairment Profile scores range from 0 (no impairment) to 4 (severe impairment). All patients underwent swallowing evaluation via VFS testing. Analyses were conducted on fluoroscopic images of 


\section{Table 1: Demographics, baseline characteristics and swallowing functions}

\begin{tabular}{|c|c|}
\hline Variables & Mean \pm SD \\
\hline Number & 56 \\
\hline \multicolumn{2}{|l|}{ Demographics } \\
\hline Age(years) & $63.02 \pm 8.20$ \\
\hline Gender (male/female) & $25 / 31(44.6 \% / 55.4 \%)$ \\
\hline Years of education(years) & $10.98 \pm 4.01$ \\
\hline \multicolumn{2}{|l|}{ Disease characteristics } \\
\hline Disease duration(years) & $4.53 \pm 4.05$ \\
\hline LEDD & $255.98 \pm 248.10$ \\
\hline UPDRS part III & $16.15 \pm 8.36$ \\
\hline H \& Y stage & $1.87 \pm 0.69$ \\
\hline Stage 1 & $19(33.9 \%)$ \\
\hline Stage 2 & $19(33.9 \%)$ \\
\hline Stage 2.5 & $13(23.2 \%)$ \\
\hline Stage 3 & $5(8.9 \%)$ \\
\hline \multicolumn{2}{|l|}{ General mental status } \\
\hline K-MMSE & $27.48 \pm 2.60$ \\
\hline CDR-SB & $0.69 \pm 1.26$ \\
\hline \multicolumn{2}{|l|}{ Swallowing function } \\
\hline Lip closure & 0 \\
\hline Hold position/tongue control & $0.16 \pm 0.37$ \\
\hline Bolus preparation/mastication & $0.13 \pm 0.42$ \\
\hline Bolus transport/lingual motion & $0.73 \pm 0.98$ \\
\hline Oral residue & $1.21 \pm 0.66$ \\
\hline Initiation of the pharyngeal swallow & $0.98 \pm 0.86$ \\
\hline Soft palate elevation & 0 \\
\hline Laryngeal elevation & $0.07 \pm 0.26$ \\
\hline Anterior hyoid motion & $0.18 \pm 0.37$ \\
\hline Epiglottic movement & $0.46 \pm 0.52$ \\
\hline Laryngeal closure & $0.53 \pm 0.53$ \\
\hline Pharyngeal stripping wave & $0.06 \pm 0.23$ \\
\hline Pharyngeal contraction & $0.23 \pm 0.40$ \\
\hline PES opening & $0.04 \pm 0.19$ \\
\hline Tongue base retraction & $1.29 \pm 0.77$ \\
\hline Pharyngeal residue & $1.61 \pm 0.98$ \\
\hline Esophageal clearance in the upright position & $0.23 \pm 0.43$ \\
\hline Penetration and aspiration & $0.46 \pm 0.93$ \\
\hline Overall oral stage & $2.22 \pm 1.82$ \\
\hline Overall pharyngeal stage & $5.44 \pm 3.03$ \\
\hline
\end{tabular}

SD, standard deviation; LEDD, Levodopa equivalent daily dosage ${ }^{29}$; UPDRS, Unified Parkinson's Disease Rating Scale; H\&Y, Hoehn and Yahr stage; K-MMSE, Korean version of Mini-Mental State Examination; CDR-SB, Clinical dementia rating-sum of box

UPDRS scores were obtained in on-state.

patients swallowing either three $\mathrm{ml}$ of thin liquid, three $\mathrm{ml}$ of thick liquid (liquid barium), or a piece of biscuit. Of the above mentioned 17 swallowing characteristics, bolus transport/lingual motion, oral residue, and tongue base retraction were analyzed using the thick liquid images; bolus preparation/mastication and pharyngeal residue were analyzed using the biscuit images; and the remainder were analyzed using the thin liquid images. Swallowed materials were optimized to evaluate each specific swallowing characteristic.

\section{Statistical Analyses}

Statistical analyses were performed with SPSS Statistics 18.0 (SPSS, Chicago, IL). Spearman partial correlation analysis was used to explore correlations of motor and neuropsychological variables with swallowing components. Age, years of education, and UPDRS III total score were included as covariates in the correlation analysis for swallowing components with raw scores of neuropsychological variables. Age and disease duration were included as covariates in the correlation analysis between swallowing components and motor variables. The significance level was set at $p<0.05$. We assumed that each domain of cognitive functions was statistically independent and the adjusted p-values were corrected by Bonferroni's method among the variables within each domain.

\section{RESULTS}

Demographic information, baseline characteristics, and the each scores of swallowing functions are shown in Table 1.

\section{Correlation Analysis Between Motor Subsets and Swallowing Components}

The results of correlation analysis between motor score and swallowing component, with age and disease duration controlled for, is described in Table 2. The total UPDRS III score was significantly associated with oral residue $(p<0.01)$ and tongue base retraction $(p=0.01)$. Bradykinesia was significantly associated with oral residue $(p<0.01)$. The subscores of tremor, rigidity, and axial impairment did not significantly correlate with any swallowing components.

\section{Correlation Analysis Between Neuropsychological Profiles and Swallowing Components}

The correlation analysis between neuropsychological measures and swallowing components, after controlling for age, years of education, and UPDRS III total score revealed significant associations between oral phase swallowing components and frontal/ executive and learning/memory functions (Table 3). During the oral phase, bolus preparation/mastication, bolus transport/lingual motion, initiation of pharyngeal swallowing, and overall oral stage negatively correlated with various cognitive domains. Initiation of pharyngeal swallowing was mildly associated with the K-MMSE score $(p=0.04)$. Bolus preparation/mastication was moderately correlated with scores on the SVLT-delayed recall $(p=0.04)$, and the Stroop word $(p<0.01)$ and color $(p=0.03)$ tests. Bolus transport/lingual motion showed moderate correlations with scores on the SVLT-delayed recall $(p=0.03)$, recognition $(p=0.01)$ and the Stroop word reading test $(p=0.03)$. The total score of the overall oral stage showed mild correlation with scores on the K-MMSE $(p=0.04)$, and moderate associations with SVLTdelayed recall $(p=0.02)$, recognition $(p=0.02)$, and the Stroop word reading test $(p<0.01)$. During the pharyngeal phase, scores on the Stroop word test were moderately associated with laryngeal 
Table 2: Correlation between phenotype and severity of motor symptoms, and swallowing components (r)

\begin{tabular}{|c|c|c|c|c|c|c|}
\hline & \multicolumn{5}{|c|}{ UPDRS score } & \multirow[b]{2}{*}{ H \& Y stage } \\
\hline & Tremor & Rigidity & Bradykinesia & Axial impairment & Total & \\
\hline \multicolumn{7}{|l|}{ Oral stage } \\
\hline \multicolumn{7}{|l|}{ Lip closure } \\
\hline \multicolumn{7}{|c|}{ Hold position/tongue control } \\
\hline \multicolumn{7}{|c|}{ Bolus preparation/mastication } \\
\hline \multicolumn{7}{|c|}{ Bolus transport/lingual motion } \\
\hline Oral residue & & & 0.394 & & 0.429 & \\
\hline \multicolumn{7}{|c|}{ Initiation of the pharyngeal swallow } \\
\hline \multicolumn{7}{|l|}{ Overall oral stage } \\
\hline \multicolumn{7}{|l|}{ Pharyngeal stage } \\
\hline \multicolumn{7}{|l|}{ Soft palate elevation } \\
\hline \multicolumn{7}{|l|}{ Laryngeal elevation } \\
\hline \multicolumn{7}{|l|}{ Anterior hyoid motion } \\
\hline \multicolumn{7}{|l|}{ Epiglottic movement } \\
\hline \multicolumn{7}{|l|}{ Laryngeal closure } \\
\hline \multicolumn{7}{|c|}{ Pharyngeal stripping wave } \\
\hline \multicolumn{7}{|l|}{ Pharyngeal contraction } \\
\hline \multicolumn{7}{|l|}{ PES opening } \\
\hline Tongue base retraction & & & & & 0.341 & \\
\hline \multicolumn{7}{|l|}{ Pharyngeal residue } \\
\hline \multicolumn{7}{|l|}{ Esophageal clearance } \\
\hline \multicolumn{7}{|l|}{ Penetration, aspiration } \\
\hline Overall pharyngeal stage & & & & & & \\
\hline
\end{tabular}

$\mathrm{r}=$ Spearman partial correlation coefficient adjusted by age and disease duration.

Values in italics, $P<.05 ; P$ values were corrected by Bonferroni's method due to multiple testing.

UPDRS score, The motor portion of the Unified Parkinson's Disease Rating Scale; H \& Y, Hoehn and Yahr; PES, pharyngo-esophageal segment

elevation $(p<0.01)$, anterior hyoid motion $(p=0.05)$ and the pharyngeal stripping wave $(p<0.01)$. Overall, higher motor and lower cognitive scores (denoting greater impairment) were associated with worse swallowing function.

\section{Discussion}

We investigated the association between swallowing components and cognitive functions assessed by neuropsychological profiles and motor signs in patients with PD. The most significant correlations were found between frontal/executive function, memory functions, and the oral phase, though a minor component of the pharyngeal phase was associated with frontal functions. In addition, motor functions correlated with oral residue and tongue base retraction.

Among the cognitive dysfunctions in PD, attention has been associated with the anticipatory phase that precedes the oral phases, which involves preparation and introduction of nutritional materials to the oral cavity. ${ }^{26}$ Another study exploring the relationship between swallowing and cognitive function revealed a moderate positive correlation between swallowing speed and cognition, although cognitive function was measured by a simple screening tool. ${ }^{6}$ Thus, cognitive decline is associated with, and may contribute to, dysphagia in in patients with PD. However, the association between individual aspects of cognition, defined by detailed neuropsychological profiles, and swallowing functions had not been adequately studied.

We evaluated the associations between cognitive functions, as assessed by detailed neuropsychological profiling, and objective swallowing measurements. We found that learning and memory functions assessed by the SVLT, and frontal executive functions assessed by the COWAT and Stroop tests, correlated to varying degrees with bolus preparation and transport processes of the oral phase. On the other hand, cognitive function was much less influential during the pharyngo-esophageal phases, which is believed to be primarily reflexive, as it is during the oral phase. We hypothesize that impaired cognitive function, particularly frontal/ executive and memory function, affect the capacity to coordinate planning and organized movements needed for mastication and lingual motion. This leads to dysphagia and aspiration in patients with PD. At the same time, the pharyngo-esophageal phases seem less likely to require, or be affected by, cognitive influences due to their non-volitionally controlled nature. ${ }^{10,27}$

The relationship between motor dysfunction and swallowing functions is also not well defined. Indeed, the question of whether disease stage or severity is an indicator of dysphagia is a controversy in the field. Leopold et al. ${ }^{17}$ reported that an advanced PD group had more abnormalities in the prepharyngeal phase, while Ali et al. ${ }^{28}$ failed to find any correlation between PD severity and 


\begin{tabular}{|c|c|c|c|c|c|c|c|c|c|c|c|c|c|c|}
\hline & \multicolumn{2}{|c|}{ General mental } & \multirow{3}{*}{$\begin{array}{c}\text { Visuospatial } \\
\text { Clock drawing } \\
\end{array}$} & \multirow{2}{*}{\multicolumn{2}{|c|}{$\begin{array}{c}\text { Attention } \\
\text { Digit span }\end{array}$}} & \multirow{3}{*}{$\begin{array}{c}\text { Language } \\
\text { BNT } \\
\end{array}$} & \multirow{2}{*}{\multicolumn{3}{|c|}{$\begin{array}{c}\text { Learning \& memory } \\
\text { SVLT }\end{array}$}} & \multicolumn{4}{|c|}{ Frontal/executive function } & \multirow[b]{3}{*}{ interference } \\
\hline & \multirow[t]{2}{*}{ MMSE } & \multirow[t]{2}{*}{ CDR SB } & & & & & & & & & VAT & & & \\
\hline & & & & $\mathbf{F}$ & B & & immediate & delayed & recognition & semantic & phonemic & word & color & \\
\hline \multicolumn{15}{|l|}{ Oral stage } \\
\hline \multicolumn{15}{|l|}{ Lip closure } \\
\hline \multicolumn{15}{|l|}{ Hold position/tongue control } \\
\hline Bolus preparation/mastication & & & & & & & & -0.330 & & & & -0.569 & -0.348 & \\
\hline Bolus transport/lingual motion & & & & & & & & -0.334 & -0.383 & & & -0.348 & & \\
\hline \multicolumn{15}{|l|}{ Oral residue } \\
\hline Initiation of the pharyngeal swallow & -0.270 & & & & & & & & & & & & & \\
\hline Overall oral stage & -0.274 & & & & & & & -0.356 & -0.358 & & & -0.493 & & \\
\hline \multicolumn{15}{|l|}{ Pharyngeal stage } \\
\hline \multicolumn{15}{|l|}{ Soft palate elevation } \\
\hline Laryngeal elevation & & & & & & & & & & & & -0.398 & & \\
\hline \multicolumn{15}{|l|}{ Anterior hyoid motion } \\
\hline \multicolumn{15}{|l|}{ Epiglottic movement } \\
\hline \multicolumn{15}{|l|}{ Laryngeal closure } \\
\hline Pharyngeal stripping wave & & & & & & & & & & & & -0.498 & & \\
\hline \multicolumn{15}{|l|}{ Pharyngeal contraction } \\
\hline \multicolumn{15}{|l|}{ PES opening } \\
\hline \multicolumn{15}{|l|}{ Tongue base retraction } \\
\hline \multicolumn{15}{|l|}{ Pharyngeal residue } \\
\hline \multicolumn{15}{|l|}{ Esophageal clearance } \\
\hline \multicolumn{15}{|l|}{ Penetration, aspiration } \\
\hline Overall pharyngeal stage & & & & & & & & & & & & & & \\
\hline
\end{tabular}

$\mathrm{r}=$ Spearman partial correlation coefficient adjusted by age, years of education and UPDRSIII total score.

Values in italics, $P<.05 ; P$ values were corrected by Bonferroni's method due to multiple testing.

MMSE, Mini-Mental State Examination; CDRSB, Clinical Dementia Rating - sum of box; BNT, Boston Naming Test; SVLT, Seoul Verbal Learning Test; COWAT, Controlled Oral Word

Association Test; F, forward; B, backward, PES, pharyngo-esophageal segment 
dysphagia. Previous work suggests that patients classified as postural instability/gait disorder type have greater swallowing impairments than those as tremor dominant type of PD. This supports the hypothesis that swallowing may be associated with more axial motor disturbances. ${ }^{6}$ Our study identified that bradykinesia was significantly associated with oral residue, as was the total UPDRS score (which was also associated with impaired tongue base retraction). These changes would be likely to produce hypokinesia of the oropharyngeal muscles. We hypothesize that as the motor symptoms progress, swallowing dysfunction spreads to the pharyngeal phase as well as the oral phase. However, the $\mathrm{H}$ \& Y stage did not correlate with any components of swallowing. Severely dysphagic subjects or patients with advanced stage PD were not included because of the potential risks during the swallowing test. In fact, our sample clustered around the early to middle stages of PD. Therefore, examination of the progression of PD and its relation to changes in swallowing dysfunction across different stages is required to confirm and extend our results.

Our study was limited by its purely correlational nature, i.e., we could not make directional attributions or establish causality. Thus, the results and their interpretation should be treated with caution.

\section{CONCLUSIONS}

In conclusion, the present finding suggests that cognitive dysfunctions, especially frontal/executive and learning/memory functions, are mainly associated with the oral phase of swallowing in patients with early stage PD. Considering that most treatments of dysphagia focus on rehabilitative training, which involves a cognitive load for success, it is important to recognize and understand cognitive influences on swallowing dysfunction in patients with PD.

\section{ACKNOWLEDGMents}

We thank the members of the KOJYP study group (Wooyoung Jang, Eungseok Oh, Jinse Park) for special comments on our work. This work was supported by a Samsung Medical Center grant (SMO1131541 and CB13152) and Soonchunhyang University Research Fund.

\section{Statement of Authorship}

JSK, JY, and JWC conceived the study, participated in the study's design and coordination, performed the statistical analysis, and drafted the manuscript. MKS and JC conducted the swallowing and neuropsychological tests. TEK assisted in the acquisition of data. All authors read and approved the final manuscript.

\section{Disclosures}

Ji Sun Kim, Jinyoung Youn, Mee Kyung Suh, Tae-Eun Kim, Juhee Chin, Jin Whan Cho have nothing to disclose. The authors declare that they have no competing interests.

\section{REFERENCES}

1. Nakashima K, Maeda M, Tabata M, Adachi Y, Kusumi M, Ohshiro H. Prognosis of Parkinson's Disease in Japan. Eur Neurol. 1997;38(Suppl 2):60-3.

2. Troche MS, Sapienza CM, Rosenbek JC. Effects of bolus consistency on timing and safety of swallow in patients with Parkinson's disease. Dysphagia. 2008;23:26-32.
3. Pfeiffer RF. Gastrointestinal dysfunction in Parkinson's disease. Parkinsonism Relat Disord. 2011;17:10-5

4. Kalf JG, de Swart BJ, Bloem BR, Munneke M. Prevalence of oropharyngeal dysphagia in Parkinson's disease: a meta-analysis. Parkinsonism Relat Disord. 2012;18:311-5.

5. Volonté MA, Porta M, Comi G. Clinical assessment of dysphagia in early phases of Parkinson's disease. Neurol Sci. 2002;23:S121-2.

6. Miller N, Allcock L, Hildreth AJ, Jones D, Noble E, Burn DJ. Swallowing problems in Parkinson disease: frequency and clinical correlates. J Neurol Neurosurg Psychiatry. 2009;80:1047-9.

7. Cereda E, Cilia R, Klersy C, et al. Swallowing disturbances in Parkinson's disease: a multivariate analysis of contributing factors. Parkinsonism Relat Disord. 2014;20:1382-7.

8. Monte FS, da Silva-Junior FP, Braga-Neto P, Nobre e Souza MA, de Bruin VM. Swallowing abnormalities and dyskinesia in Parkinson's disease. Mov Disord. 2005;20:457-62.

9. Lam K, Lam FK, Lau KK, et al. Simple clinical tests may predict severe oropharyngeal dysphagia in Parkinson's disease. Mov Disord. 2007;22:640-4.

10. Steele CM, Miller AJ. Sensory input pathways and mechanisms in swallowing: a review. Dysphagia. 2010;25:323-33.

11. Miller AJ. Characteristics of the swallowing reflex induced by peripheral nerve and brain stem stimulation. Exp Neurol. 1972;34:210-22.

12. Hawkes CH, Del Tredici K, Braak H. A timeline for Parkinson's disease. Parkinsonism Relat Disord. 2010;16:79-84.

13. Grinberg LT, Rueb U, Alho AT, Heinsen H. Brainstem pathology and non-motor symptoms in PD. J. Neurol Sci. 2010;289:81-8.

14. Suntrup S, Teismann I, Bejer J, et al. Evidence for adaptive cortical changes in swallowing in Parkinson's disease. Brain. 2013; 136(Pt 3):726-38.

15. Hamdy S, Mikulis DJ, Crawley A, et al. Cortical activation during human volitional swallowing: an event-related fMRI study. Am J Physiol. 1999;277:G219-25.

16. Umemoto G, Tsuboi Y, Kitashima A, Furuya H, Kikuta T. Impaired food transportation in Parkinson's disease related to lingual bradykinesia. Dysphagia. 2011;26:250-5.

17. Leopold NA, Kagel MC. Prepharyngeal dysphagia in Parkinson's disease. Dysphagia. 1996;11:14-22.

18. Takahashi T, Miyamoto T, Terao A, Yokoyama A. Cerebral activation related to the control of mastication during changes in food hardness. Neuroscience. 2007;145:791-4.

19. Minato A, Ono T, Miyamoto JJ, Honda E, Kurabayashi T, Moriyama K. Preferred chewing side-dependent two-point discrimination and cortical activation pattern of tactile tongue sensation. Behav Brain Res. 2009;203:118-26.

20. Brodsky MB, McNeil MR, Martin-Harris B, Palmer CV, Grayhack JP, Abbott KV. Effects of divided attention on swallowing in healthy participants. Dysphagia. 2012;27:307-17.

21. Kang Y, Na DL, Hahn S. A validity study on the Korean MiniMental State Examination (K-MMSE) in dementia patients. J Korean Neurol Assoc. 1997;15:300-8.

22. Uc EY, McDermott MP, Marder KS, et al. Incidence of and risk factors for cognitive impairment in an early Parkinson disease clinical trial cohort. Neurology. 2009;73:1469-77.

23. Kang Y, Na DL. Seoul Neuropsychological Screening Battery. Incheon: Human Brain Research \& Consulting Co; 2003.

24. Kim H, Na DL. Normative Data on the Korean Version of the Boston Naming Test. J Clin Exp Neuropsychol. 1999;21:127-33.

25. Martin-Harris B, Brodsky MB, Michel Y, et al. MBS measurement tool for swallow impairment-MBSImp: establishing a standard. Dysphagia. 2008;23:392-405.

26. Brodsky MB, Verdolini Abbott $\mathrm{K}$, McNeil MR, Palmer CV, Grayhack JP, Martin-Harris B. Effects of divided attention on swallowing in persons with idiopathic Parkinson's disease. Dysphagia. 2012;27:390-400.

27. Peyron M-A, Gierczynski I, Hartmann C, et al. Role of physical bolus properties as sensory inputs in the trigger of swallowing. PLoS One. 2011;6:e21167.

28. Ali GN, Wallace KL, Schwartz R, Decarle DJ, Zagami AS, Cook IJ. Mechanisms of oral-pharyngeal dysphagia in patients with Parkinson's Disease. Gastroenterology. 1996;110:383-92. 